\title{
Comparação de duas estratégias de desempenho para avaliação da velocidade crítica de caminhada
}

\section{Comparison of two strategies for assessing critical walking velocity}

Leandro Quadro Corrêa ${ }^{1}$ Airton José Rombaldi ${ }^{1}$

Eduardo Kokubun 2

Paula Aver Bretanha Ribeiro 2

1 Universidade Federal de Pelotas. Pelotas, RS. Brasil

2 Universidade Estadual Paulista Campus de Rio Claro. Rio Claro, SP. Brasil

Recebido em 19/05/08 Revisado em 21/11/08 Aprovado em 01/12/08
Resumo - O objetivo deste estudo foi comparar duas estratégias de desempenho em testes de caminhada, tempo fixo com intensidade autoselecionada e ritmo fixo, na determinação dos parâmetros da Velocidade Crítica de Caminhada (VCC). Fizeram parte da amostra 14 voluntárias (idade $=60,8 \pm 10,3$ anos), submetidas aleatoriamente a três testes de caminhada de tempo fixo (3, 6, e 9 minutos) e três testes com intensidade fixa, que variaram entre 10 e 15 segundos a cada 20 metros. Estes testes preditivos foram utilizados para o cálculo da VCC. O erro padrão da estimativa da VCC apresentou valor médio de 4,96\% na estratégia de ritmo fixo e 2,98\% na estratégia de tempo fixo, o que representa boa estimativa deste parâmetro. Os resultados apontaram alta correlação entre as estratégias de coleta para a VCC $(r=0,73 ; p<0,01)$. A média dos coeficientes de determinação para o modelo de ritmo fixo foram $R^{2}=0,98 \pm 0,03$ e para tempo fixo $R^{2}=0,99 \pm 0,002$. Os resultados da ANCOVA para os testes preditivos demonstraram não haver diferença entre as estratégias de coleta $(\mathrm{p}=0,38)$, na comparação dos sujeitos x estratégias $(\mathrm{p}=0,29)$ e nas estratégias $\mathrm{x}$ tempo $(\mathrm{p}=0,26)$. A regressão geométrica, comparando as estratégias tempo fixo $\mathrm{x}$ ritmo fixo $(1,42 \pm 0,14$ e 1,38 $\pm 0,21 \mathrm{~m} / \mathrm{s})$ também não apontou diferenças, assim como o método de Bland \& Altman. Concluiu-se que as duas estratégias foram equivalentes na avaliação do desempenho na amostra estudada.

Palavras-chave: Desempenho atlético; Limiar anaeróbio; Mulheres; Avaliação.

Abstract - The aim of this study was to compare two strategies of walking test performance (self-paced intensity and fixed pace) for the determination of critical walking velocity (CWV) parameters. Fourteen female volunteers (age $=60.8 \pm 10.3$ years) were randomly submitted to three fixed-time walking tests (3, 6 and 9 minutes) and three fixed-pace tests ranging from 10 to 15 seconds at intervals of 20 meters. These predictive tests were used to calculate CWV. The mean standard error of the CWV estimate was 4.96\% for the fixed-pace strategy, and 2.98\% for the fixed-time strategy, corresponding to an adequate estimation. The results showed a high correlation between strategies $(r=0.73 ; p<0.01)$. The mean coefficient of determination was $R^{2}=$ $0.98 \pm 0.03$ for the fixed-pace model and $R^{2}=0.99 \pm 0.002$ for the self-paced model. ANCOVA for the predictive tests showed no differences between strategies $(p=0.29)$, subjects $x$ strategies $(p=0.29)$, or strategies $x$ time $(p=0.26)$. Geometric regression comparing the fixed-time $x$ fixedpace strategies $(1.42 \pm 0.14$ and $1.38 \pm 0.21 \mathrm{~m} / \mathrm{s})$ or the Bland-Altman method revealed no differences between performance strategies. We conclude that the two strategies were equivalent for the evaluation of performance in the sample studied.

Key words: Athletic performance; Anaerobic thereshold; Women; Evaluation. 


\section{INTRODUÇÃO}

A procura de alternativas que facilitem a avaliação, a prescrição e o acompanhamento do desempenho físico, em populações especiais, tem instigado os pesquisadores na tentativa de contribuir para a prescrição apropriada e individualizada de exercícios físicos. Testes de caminhada de tempo fixo e intensidade auto-selecionada com duração entre 2 e 12 minutos têm sido utilizados para a avaliação da capacidade cardiorrespiratória, em diferentes populações, tendo em vista sua fácil aplicação, baixo custo e alta correlação com capacidade funcional de pacientes ${ }^{1}$.

O modelo de avaliação da Potência Crítica (Pcrit) proposto por Monod \& Scherrer ${ }^{2}$, permite avaliar a capacidade aeróbia e anaeróbia, simultaneamente, e tem se apresentado como uma boa estratégia de avaliação em ambiente laboratorial e de campo ${ }^{3}$. Teoricamente, a intensidade referente à Pcrit corresponde a mais alta intensidade em que o exercício poderia ser realizado por tempo prolongado. Acima dessa intensidade, haveria a utilização de um estoque limitado de energia anaeróbia para atender as necessidades adicionais do esforço. $\mathrm{O}$ esgotamento desse estoque, denominado capacidade de trabalho anaeróbio (CTA), levaria o executante à exaustão ${ }^{4,5}$. Para alguns autores, a Pcrit equivale ao limiar anaeróbio determinado pela concentração fixa de $4 \mathrm{mM}$ de lactato sanguíneo ou ao máximo estado estável de lactato ${ }^{7,8}$. Este modelo exige testes retangulares exaustivos e utilização de cargas preditivas de intensidades controladas, o que limita, em parte, sua aplicação, embora já tenha sido testado em pacientes com doença pulmonar obstrutiva crônica (DPOC) $)^{9-11}$ e idosos ${ }^{12}$.

Classicamente, a Pcrit é calculada por meio de testes preditivos com intensidade fixa, medindose o tempo até a exaustão. Estudos anteriores já demonstraram que o modelo da Pcrit pode ser aplicado em exercícios que envolvem deslocamentos, como corrida, natação, remo entre outras atividades, nos quais a intensidade é proporcional à velocidade ${ }^{13-16}$.

Casas et al..$^{10}$ aplicaram o modelo de Pcrit em pacientes com DPOC, fixando os testes preditivos em 90, 95, 100 e 105\% do consumo máximo de oxigênio. Os resultados demonstraram bons ajustes e alta correlação com a capacidade funcional dos pacientes. Entretanto, testes de caminhada, comumente realizados em ambulatórios ou corredores de hospitais, utilizam avaliações com tempo fixo e intensidade auto-selecionada, nas quais o sujeito desempenha de acordo com sua capacidade, o que possibilita a avaliação de sujeitos com limitações a esforços máximos.

Levando-se em conta que a caminhada é a atividade física mais praticada pelos brasileiros ${ }^{17}$, a avaliação e a identificação da intensidade adequada podem beneficiar muitos indivíduos para que realizem de maneira mais eficiente seus programas de caminhadas; neste sentido, o objetivo do presente estudo foi comparar as estimativas de velocidade crítica de caminhada (VCC - parâmetro aeróbio) e capacidade de caminhada anaeróbia (CCA - parâmetro anaeróbio) obtidas a partir de duas estratégias de desempenho: (1) testes de caminhada de tempo fixo com intensidade auto-selecionada e (2) testes de ritmo fixo.

\section{PROCEDIMENTOS METODOLÓGICOS}

Participaram deste estudo 14 voluntárias frequentadoras de um projeto de Exercícios Físicos para diabéticos, hipertensos e obesos da Universidade Federal de Pelotas. Todas possuíam liberação médica para a prática de exercícios físicos e eram fisicamente ativas há mais de seis meses. Elas foram informadas previamente dos procedimentos do estudo e assinaram um termo de consentimento livre e esclarecido para participação. $O$ protocolo do presente estudo foi avaliado e aprovado pelo Comitê de Ética em Pesquisa (processo número 183/2005).

\section{Antropometria}

A massa corporal total foi medida com as voluntárias em pé, no centro da plataforma, de costas para a escala, e olhando para um ponto fixo à sua frente, de modo que evitasse oscilações na leitura. A estatura foi medida com as avaliadas em pé, eretas, com os pés unidos, calcanhares, nádegas, costas e parte posterior da cabeça em contato com a escala. A medida foi tomada da planta dos pés até o vórtex cerebral, com a cabeça voltada para o plano de Frankfurt.

Para a realização destas medidas utilizou-se uma balança digital com estadiômetro da marca Soehnle 7755 , com precisão máxima para massa corporal de 200 kg e mínima de 100 g, e com precisão de 0,1 centímetro para estatura. Os valores foram utilizados, posteriormente, para o cálculo do índice de massa corporal (IMC).

\section{Protocolo experimental}

- Familiarização

Foram empregados testes de caminhada, em mo- 
delo vai-vem de 20 metros, em superfície plana, nos quais, inicialmente, os sujeitos realizaram um teste com velocidade auto-selecionada para familiarização, sendo instruídos a caminharem na maior velocidade possível durante 6 minutos.

Testes de caminhada: as voluntárias foram submetidas à primeira etapa dos testes preditivos do modelo de avaliação da VCC, quando foram realizados três testes de caminhada de tempo fixo (3, 6, e $9 \mathrm{~min})$, em intensidade auto-selecionada, em vai-vem de $20 \mathrm{~m}$, em ordem randomizada, e em diferentes dias, no prazo de uma semana, medindose a distância percorrida.

Após a realização dos testes de tempo fixo, os sujeitos foram submetidos a mais três testes, no mesmo modelo vai-vem de 20 metros, porém com ritmo fixo de caminhada individualizado, com tempos que variaram entre 10 e 15 segundos a cada 20 metros, e que levassem a exaustão entre um e 10 minutos. Foi utilizado sinal sonoro, a cada 20 metros, para que os participantes acompanhassem o ritmo necessário.

Para cada um dos testes, foram coletados tempo limite e distância percorrida para o dado tempo para determinação dos parâmetros da Pcrit. Para delimitação dos espaços dos testes, foram utilizados cones coloridos para facilitar a visualização. Foi traçada, no piso, uma linha localizada a uma distância de um metro a frente de cada um dos cones de demarcação do percurso. A cada tentativa que o sujeito não se localizasse dentro desse limite de tolerância era considerado um erro, o terceiro erro consecutivo determinava o fim do teste. Além disso, os participantes poderiam desistir do teste, voluntariamente, se não sentissem mais condições de continuar, mesmo que não tivessem cometido nenhum erro.

\section{Tratamento estatístico}

- Cálculos dos parâmetros da Pcrit

O cálculo dos parâmetros aeróbio (inclinação da reta) e anaeróbio (intercepto da regressão no eixo y) do modelo de Pcrit ${ }^{2}$ foi feito a partir de regressão linear simples. Neste caso, a potência foi substituída por distâncias e tempos, fornecendo parâmetros de VCC para o parâmetro aeróbio e CCA para o parâmetro anaeróbio. Para o cálculo da VCC foram utilizados as distâncias $\left(D_{\text {Lim }}\right)$ e os tempos-limite $\left(\mathrm{T}_{\text {Lim }}\right)$ dos testes e utilizou-se a equação da regressão linear: $\mathrm{D}_{\text {Lim }}=\mathrm{CCA}+\mathrm{VCC} \cdot \mathrm{T}_{\mathrm{Lim}}$

Os dados descritivos foram apresentados, em média, com seus respectivos desvios-padrão (DP). Para testar a equivalência dos modelos de coleta foi utilizada análise de covariância (ANCOVA), na qual a distância limite foi definida como variável dependente, o sujeito e a condição (tempo fixo x ritmo fixo) como variáveis independentes, e o tempo $\left(\mathrm{T}_{\text {LIM }}\right)$ como covariável. O Intervalo de Confiança $\left(\mathrm{IC}_{95 \%}\right)$ foi utilizado para avaliar cada parâmetro calculado. Para comparação das estratégias de coleta foi utilizada a análise de regressão geométrica proposta por Ludbrock ${ }^{18} \mathrm{e}$ o método de Bland \& Altman ${ }^{19}$ para avaliar a concordância entre elas. O nível de significância adotado para todas as análises foi $\mathrm{p}<0,05$.

\section{RESULTADOS}

As médias de idade, peso, estatura e IMC das voluntárias foram respectivamente $60,8 \pm 10,3$ anos, 71,8 $\pm 12,7 \mathrm{~kg}, 1,6 \pm 0,1$ metros e $29,3 \pm 4,5 \mathrm{~kg} / \mathrm{m}^{2}$.

Os testes preditivos foram classificados como leves, moderados e vigorosos, de acordo com a velocidade média desenvolvida, sendo os mais longos considerados leves e os mais curtos como vigorosos. Os resultados das distâncias percorridas, dos tempos e respectivas amplitudes estão apresentados nas Tabelas 1 e 2.

Tabela 1. Médias, desvios-padrão e amplitudes dos tempos e distância percorrida nos testes preditivos com a estratégia de ritmo fixo.

\begin{tabular}{lcc}
\hline Intensidades & Tempos $(\mathrm{s})$ & Distâncias $(\mathrm{m})$ \\
\hline Leve & & \\
Média $\pm \mathrm{dp}$ & $551,5 \pm 176,0$ & $816,9 \pm 276,3$ \\
Amplitude & $138-688$ & $220-1040$ \\
Moderado & & \\
Média $\pm d p$ & $209,0 \pm 127,4$ & $321,4 \pm 202,2$ \\
Amplitude & $70-520$ & $100-800$ \\
Intenso & & \\
Média $\pm d p$ & $139,2 \pm 79,4$ & $221,4 \pm 123,2$ \\
Amplitude & $66-324$ & $120-540$ \\
\hline
\end{tabular}

Tabela 2. Tempos, médias, desvio-padrão e amplitude das distâncias percorridas nos testes preditivos com a estratégia de tempo fixo.

\begin{tabular}{lcc}
\hline Intensidades & Tempos $(\mathrm{s})$ & Distâncias $(\mathrm{m})$ \\
\hline Leve: & & \\
Média \pm DP & 540,0 & $610-860$ \\
Amplitude & & \\
Moderada: & & $519,8 \pm 63,2$ \\
Média \pm DP & 360,0 & $420-600$ \\
Amplitude & & \\
Intensa: & & $267,9 \pm 30,1$ \\
Média \pm DP & 180,0 & $215-300$ \\
Amplitude & & \\
\hline
\end{tabular}

A velocidade média nos testes preditivos foi expressa em percentuais em relação à VCC calculada 
nas respectivas estratégias de coletas. A existência de valores inferiores a $100 \%$ pode ser interpretada como uma evidência de que o ajuste foi inadequado; desta maneira, todas as cargas preditivas devem ser realizadas em intensidade superior a $100 \%$ da VCC. Os testes que não preencheram esse requisito foram excluídos. Os dados utilizados para a estimativa do modelo da VCC estão apresentados na Tabela 3.

Tabela 3. Médias, desvios-padrão e erros-padrão das estimativas dos parâmetros da velocidade crítica (VCC e CCA) nas estratégias de ritmo fixo e tempo fixo.

\begin{tabular}{|c|c|c|c|c|c|c|}
\hline & \multicolumn{3}{|c|}{ Ritmo fixo } & \multicolumn{3}{|c|}{ Tempo fixo } \\
\hline & Leve & $\begin{array}{c}\text { Mode- } \\
\text { rado }\end{array}$ & Intenso & Leve & $\begin{array}{c}\text { Mode- } \\
\text { rado }\end{array}$ & Intenso \\
\hline Média & 106,3 & 108,6 & 116,4 & 103,4 & 106,2 & 109,7 \\
\hline DP & 11,1 & 4,8 & 8,5 & 2,2 & 4,3 & 6,4 \\
\hline Máximo & 141,7 & 117,9 & 132,1 & 108,0 & 115,6 & 123,5 \\
\hline Mínimo & 101,1 & 104,3 & 107,8 & 100,7 & 100,0 & 102,0 \\
\hline
\end{tabular}

Os resultados da Tabela 3 demonstram que em todas as intensidades os valores foram superiores a 100\%, demonstrando a adequação do ajuste.

Os resultados da tabela 4 demonstram que o erro padrão da estimativa (EPE) para a VCC atende ao critério de $10 \%$ proposto por $\mathrm{Hill}^{3}$ e os valores médios foram de 4,96\% na estratégia de ritmo fixo e 2,94\% na estratégia de tempo fixo, o que representa boa estimativa deste parâmetro. $\bigcirc$ mesmo não aconteceu com a CCA em nenhuma das estratégias, nas quais os valores médios foram superiores a 10\% em ambas as estratégias de coleta.

Tabela 4. Apresenta as médias, os desvios-padrão e erros padrões das estimativas dos parâmetros da velocidade crítica (VCC e CCA) nas estratégias de ritmo fixo e tempo fixo.

\begin{tabular}{lccr}
\hline Testes & Parâmetro & EPE & IC 95\% \\
\hline Ritmo fixo: & & & \\
& $1,41 \pm 0,2$ & $0,07 \pm 0,16$ & $(1,27-1,55)$ \\
VCC $(\mathrm{m} / \mathrm{s})$ & & & \\
CCA $(\mathrm{m})$ & $28,4 \pm 24,5$ & $14,0 \pm 26,0$ & $(0,40-56,40)$ \\
Tempo fixo: & & & \\
VCC $(\mathrm{m} / \mathrm{s})$ & $1,36 \pm 0,18$ & $0,04 \pm 0,03$ & $(1,28-1,44)$ \\
$\operatorname{CCA}(\mathrm{m})$ & $24,5 \pm 13,9$ & $17,8 \pm 10,4$ & $(-11,10-60,20)$ \\
\hline
\end{tabular}

Os resultados apontaram também alta correlação entre as estratégias de coleta para a VCC ( $\mathrm{r}=$ 0,73; $\mathrm{p}<0,01)$. O mesmo não ocorreu com a CCA $(r=-0,42 ; p>0,05)$, demonstrando a diferença da estimativa do parâmetro anaeróbio para as duas metodologias de avaliação utilizadas.

No que diz respeito aos coeficientes de determinação para as estratégias de coleta, a média para o modelo de ritmo fixo foi 0,98 \pm 0,06 e para o modelo de tempo fixo 0,99 $\pm 0,002$. Estes valores indicaram excelente qualidade no ajuste.

A ANCOVA não apresentou diferenças significativas para a VCC e para a CCA entre os modelos de coleta, seja na estratégia ritmo fixo $\mathrm{x}$ estratégia de tempo fixo ( $p=0,38)$, na comparação dos sujeitos $\mathrm{x}$ estratégias de coleta $(\mathrm{p}=0,29)$ ou na comparação entre condições de coleta $x$ tempo ( $p$ =0,26). Na comparação das estratégias de coleta, não houve diferença significativa entre elas em relação à VCC, de acordo com o $\mathrm{IC}_{95 \%}$.

Utilizou-se também a análise de regressão geométrica, que conforme Ludbrook ${ }^{18}$, é o método apropriado para comparar dois métodos de medidas. Para tanto, foi necessário estimar: 1) o coeficiente angular e o intercepto da regressão linear entre as duas medidas, e 2) os intervalos de confiança para o coeficiente angular e o intercepto. Admite-se que quando o intervalo de confiança do intercepto compreende o valor zero e o de inclinação o valor um, não há diferença entre as duas formas de coleta, concluindo, dessa forma, que há erro fixo quando o intercepto não compreende o valor 0 (zero); e quando a inclinação é diferente de 1 (um) há erro proporcional. A inclinação e o intercepto da regressão geométrica da VCC, comparando o tempo fixo com o ritmo fixo, foram de 1,42 $\pm 0,14$ e 1,38 $\pm 0,21 \mathrm{~m} / \mathrm{s}$, com intervalos de confiança de 0,58 a 1,16 e $-0,27$ a 0,56 m/s para a inclinação e o intercepto, respectivamente; portanto, também não houve diferença nos modelos de coleta neste modelo de comparação.

As Figuras 1 e 2 apresentam os resultados da concordância entre os dois modelos de coleta para VCC e CCA, através do método de Bland \& Altman ${ }^{19}$, os quais demonstram haver boa concordância entre os modelos.

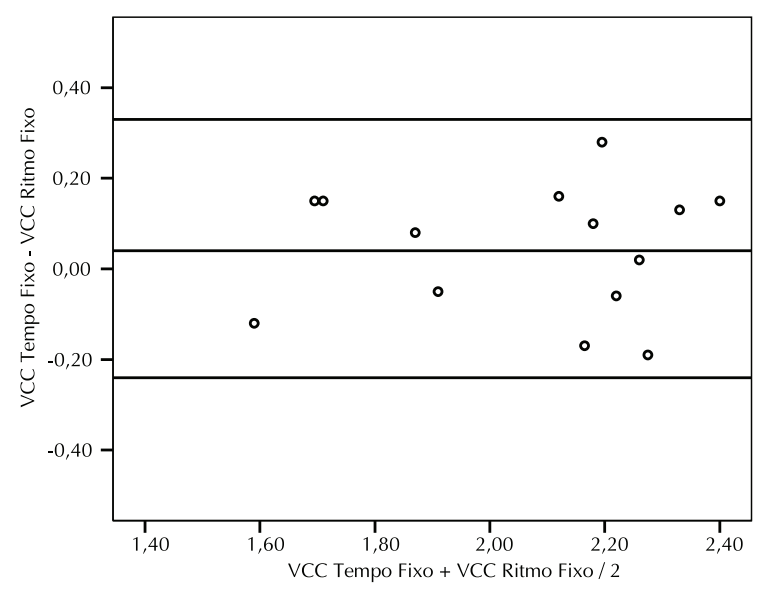

Figura 1. Concordância na VCC das duas estratégias de desempenho através do método de Bland \& Altman ${ }^{19}$. 


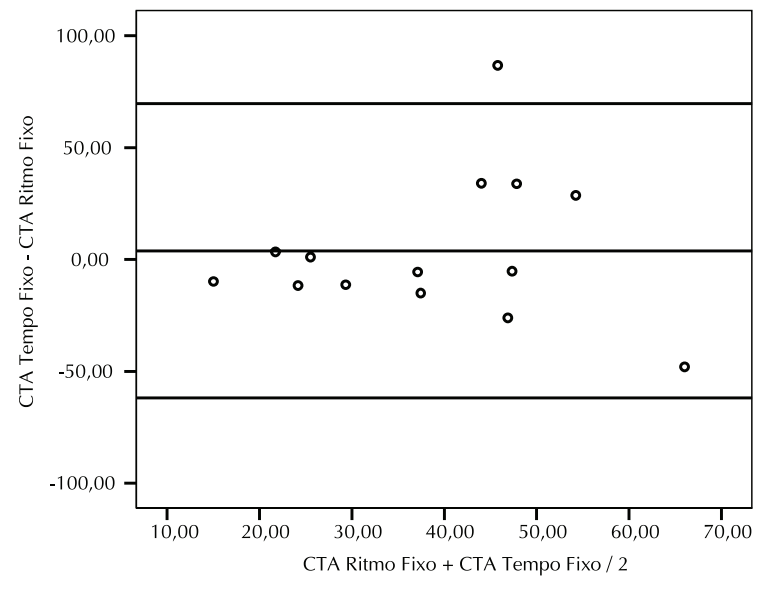

Figura 2. Concordância da CTA nas duas estratégias de coleta através do método de Bland \& Altman ${ }^{19}$.

\section{DISCUSSÃO}

O propósito do presente estudo foi comparar as estimativas de VCC e CCA obtidas a partir de duas estratégias de desempenho. Para isto, utilizou-se o modelo de avaliação no formato de vai-vem de 20 metros para determinação dos parâmetros da VCC e foi encontrada alta correlação para o parâmetro aeróbio entre as estratégias de coleta adotadas, ritmo ou tempo fixo $(r=0,73)$. O mesmo ocorreu com os coeficientes de determinação, nos quais as médias foram 0,98 $\pm 0,06$ e 0,99 $\pm 0,002$ para o modelo de ritmo fixo e para o modelo de tempo fixo, respectivamente. Estes valores indicaram excelente qualidade no ajuste.

Utilizando um modelo semelhante de desempenho, Okuno et al. ${ }^{13}$ estudaram a estratégia de vai-vem de 20 metros em corrida, na qual encontraram correlação elevada com um modelo contínuo de corrida $(\mathrm{r}=0,93 ; \mathrm{p}<0,01)$. Além disso, verificaram alta estimativa na reprodutibilidade da velocidade crítica $(r>0,90)$ e elevado coeficiente de determinação $\left(R^{2} \geq 0,98\right)$, demonstrando que $o$ modelo de velocidade crítica pode ser aplicado a este tipo de exercício.

A validade da Pcrit a diferentes modelos de exercício tem sido avaliada ${ }^{14,16,20}$. Entretanto, em estudos anteriores foram utilizados testes preditivos exaustivos e de intensidade controlada. Usualmente, os testes de Pcrit adotam a intensidade dos testes preditivos como a variável manipulada (independente) e o tempo até a exaustão como a observada (dependente).

A fim de estabelecer uma relação entre o de avaliação de Pcrit e indicadores de capacidade funcional, verificou-se a relação deste modelo com atividades da vida diária em indivíduos idosos ${ }^{12,21}$ e pacientes ${ }^{10,22}$.
Os resultados demonstraram relação com a avaliação da aptidão física em um contexto de saúde.

Para sujeitos que realizam programas de exercícios físicos, tendo a caminhada como atividade principal devido à inabilidade de praticar exercícios mais intensos, a avaliação da VCC parece ser uma estratégia plausível, por utilizar cargas retangulares com intensidades autoselecionadas, em que os indivíduos podem facilmente administrar seu desempenho.

Os resultados demonstraram que o EPE associado aos parâmetros foi inferior a 10 \% nas duas estratégias, o que indica que não determinar a intensidade de caminhada não implica perda de qualidade na estimativa dos parâmetros.

Os valores de EPE para CCA nas duas estratégias de coleta foram superiores a 10\%, não demonstrando boa estimativa para este parâmetro. O mesmo ocorreu com Papotti et al. ${ }^{15}$, em um estudo com nadadores de ambos os sexos, em que apontou-se que a CTA sofre influência das variações da energia proveniente dos sistemas anaeróbio e aeróbio, não fornecendo uma estimativa real da capacidade anaeróbia. Neste estudo, a média dos erros dos coeficientes angulares variou entre 9\% e $29 \%$, indicando que a CTA parece não ser um bom parâmetro na avaliação da aptidão anaeróbia e predição de performances em nado crawl.

A baixa correlação da CCA $(r=-0,42)$ obtida nos modelos de coleta do presente estudo também foram encontradas por Okuno et al..$^{13}$, quando compararam o modelo de corrida vai-vem com o modelo de corrida contínua $(r=0,09)$. Esses autores sugeriram que a baixa qualidade da estimativa da CCA pode ter sido devido ao modelo descontínuo de exercício, tendo em vista que o sistema de vai-vem utiliza-se de períodos de aceleração e desaceleração, o que pode ter influenciado nos resultados encontrados.

No presente estudo, utilizamos a análise de regressão geométrica para verificação da existência de possíveis erros associados às estimativas, sejam eles erros fixos ou proporcionais. Usando esse procedimento, é possível calibrar um método contra o outro, através das equações de regressão de modo que os resultados de um método podem ser comparados ou convertidos. Nossos resultados demonstraram que não existe erro fixo nem proporcional para a VCC. Portanto, as duas estratégias de coleta são equivalentes para a determinação da VCC.

Embora os resultados demonstrem similaridade entre as estratégias, uma limitação de nosso estudo foi a ausência de medidas fisiológicas, as quais poderiam esclarecer o significado fisiológico dos parâmetros estimados pelos nossos testes. 


\section{CONCLUSÃO}

Considerando que as duas estratégias de coleta se equivalem, a utilização dos testes de caminhada de intensidade autoselecionada parece ser válida para a determinação do parâmetro aeróbio do modelo de velocidade crítica de caminhada. Portanto, a similaridade entre as duas formas de coleta favorece a utilização da metodologia de tempo fixo, pela facilidade de aplicação, possibilidade de execução em massa e fácil instrução. Desta maneira, concluímos que os testes de caminhada de intensidade autoselecionada se apresentam como um modelo de avaliação válido para a população estudada. Outros estudos utilizando este modelo em vai-vem, para populações distintas, seriam de grande relevância para melhores esclarecimentos sobre a validade do modelo da VCC.

\section{REFERÊNCIAS BIBLIOGRÁFICAS}

1. Solway S, Brooks D, Lacasse Y, Thomas S. A qualitative systematic overview of the measurement properties of functional walk tests used in the cardiorespiratoru domain. Chest 2001;119(1):256-270.

2. Monod H, Scherrer J. The work capacity of a synergic muscular group. Ergonomics 1965;8(3):329-338.

3. Hill DW. The critical power concept. Sports Med 1993;16:237-254.

4. Jenkins DG, Quigley BM. Blood lactate in trained cyclists during cycle ergometry at critical power. Eur J Appl Physiol Occup Physiol 1990;61(3-4):278-283.

5. DeVries HA, Moritani T, Nagata A, Megnussen K. The relation between critical power and neuromuscular fatigue as estimated from eletromyographic data. Ergonomics 1982;25(8):783-791.

6. Lechevalier JM, Vandewalle H, Chatard JC, Moreaux A, Gandrieux V, Besson F, Monod H. Relationship between the $4 \mathrm{mmol}$ running velocity, the time-distance relationship and the Léger-Boucher's test. Arch Int Physiol Biochim Biophys 1989;97(5):355-360.

7. Smith CGM, Jones AM. The relationship between critical velocity, maximal lactate steady-state velocity and lactate turnpoint velocity in runners. Eur J Appl Physiol 2001;85(1-2):19-26.

8. Sid-Ali B, Vandewalle H, Chair K, Moreaux A, Monod $\mathrm{H}$. Lactate steady state velocity and distance-exhaustion time relationship in running. Eur J Appl Physiol Occup Physiol 1991;99(4):297-301.

9. Malaguti C, Nery LE, Corso S, Fuccio MB, Lerario $\mathrm{MC}$, Cendon S, Neder JA. Alternative strategies for exercise critical power estimation in patients with COPD. Eur J Appl Physiol 2006;96(1):59-65.

10. Casas A, Vilaro J, Rabinovich R, Mayer A, Barbera JA, Rodriguez-Roisin R, Roca J. Encouraged 6-min Walking Test Indicates Maximum Sustainable Exercise in COPD Patients. Chest 2005;128(1):55-61.
11. Neder JA, Jones PW, Nery LE, Whipp BJ. Determinants of the Exercise Endurance Capacity in Patients with Chronic Obstructive Pulmonary Disease.The PowerDuration Relationship. Am J Respir Crit Care Med 2000;162(2):497-504.

12. Capodaglio EM, Saibene FA. Test to assess the mechanical power sustainable during everyday activities in older people. Age Ageing 2003;32(1):31-36.

13. Okuno NM, Perandini LAB, Moyses EP, Nakamura PM, Ribeiro PAB, Nakamura FY. Aplicação do modelo de velocidade crítica à corrida bidirecional de 20 metros. Rev Educ Física 2007;18:50-53.

14. Nakamura FY, Borges TO, Brunetto AF, Franchini E. Correlação entre os parâmetros do modelo de potência crítica no cicloergômetro de membros superiores e no caiaque. Rev Bras Cien Mov 2005;13(2):41-47.

15. Papoti M, Zagatto AM, Júnior PBF, Cunha SA, Martins LEB, Gobatto CA. Utilização do intercepto-y na avaliação da aptidão anaeróbia e predição da performance de nadadores treinados. Rev Bras Med Esp 2005;11(2):1-5.

16. Kokubun E. Velocidade Crítica como estimadora do limiar anaeróbio na natação. Rev Paul Educ Fís 1996;10(1):5-20.

17. Hallal PC, Azevedo MR, Reichert FF, Siqueira FV, Araújo CL, Victora CG. Who, when, and how much? Epidemiology of walking in a middle-income country. Am J Prev Med 2005;28(2):156-161.

18. Ludbrook J. Comparing methods of measurement. Clin Exp Pharmacol Physiol 1997;24(2):193-203.

19. Bland JM, Altman DG. Statistical methods for assessing agreement between two methods of clinical measurement. Lancet 1986;8(1):307-310.

20. Housh DJ, Housh TJ, Bauge SM. A methodological consideration for the determination of critical power and anaerobic work capacity. Res Q Exer Sport 1990;61(4):406-409.

21. Overend TJ, Cunningham DA, Paterson DH, Smith WDF. Physiological responses of young and elderly men to prolonged exercise at critical power. Eur J Appl Physiol 1992;64(2):187-193.

22. Dolmage T, Goldstein R. The concept of critical power in the evaluation of the exercise capacity of CODP patiens. Chest 1999;16(4):243-249.

\footnotetext{
Endereço para correspondência

Airton José Rombaldi

Universidade Federal de Pelotas

Escola Superior de Educação Física

Departamento de Desportos

Rua Luís de Camões, 625. Três Vendas

96055-630 - Pelotas, RS.

E-mail: rombaldi@ufpel.tche.br
} 